\title{
Photon generation in resonator with time dependent boundary conditions
}

\author{
I. S. Lobanov ${ }^{1,2}$, A. I. Trifanov ${ }^{1}$, E. S. Trifanova ${ }^{1}$, I. Y. Popov ${ }^{1}$, E. Fedorov ${ }^{1}$, K. V. Pravdin ${ }^{1}$, M. A. Moskalenko ${ }^{1}$ \\ ${ }^{1}$ ITMO University, Kronverkskiy, 49, St. Petersburg, 197101, Russia \\ ${ }^{2}$ St. Petersburg State University, St. Petersburg, 198504, Russia \\ lobanov.igor@gmail.com, alextrifanov@gmail.com, \\ etrifanova@gmail.com,popov1955@gmail.com, fedevg95@mail.ru
}

PACS 02.30.Jr, 03.65.Ge

DOI 10.17586/2220-8054-2021-12-1-73-80

\begin{abstract}
We present a simulation of Casimir field generation in the 1D cavity with moving walls and arbitrary variation of boundary conditions. We design a numerical scheme based on the finite element method and compare photon generation due to variation of the geometry of the cavity and due to perturbation of the boundary conditions.
\end{abstract}

Keywords: dynamical Casimir effect, photon generation, numerical modelling.

Received: 19 February 2021

Revised: 23 February 2021

\section{Introduction}

Quantum parametric processes are very important mechanisms for constructing and manipulating of quantum states in a variety of aspects of quantum science and technologies. The most impressive properties of such processes are stimulation of quantum vacuum and real photon production under conditions near resonant in various quantum systems [1]. One of these is dynamical Casimir effect (DCE)- the process which demonstrates electromagnetic field generation in an empty cavity due to fast moving boundaries or nonadiabatic changing of boundary conditions or medium properties [2-6]. It was predicted in [7] and experimentally approved [8]. A comprehensive review about the theoretical models and possible implementations of DCE as soon as features of Casimir radiation photodetection one can find in $[9,10]$.

Observation of DCE is accompanied by difficulties in organization of boundary movement with the speed near the speed of light. Alternatively, fast changing of boundary conditions are used [8]. This is possible in superconducting devices like SQUID. It seems possible to realize photon generation under DCE in low-dimensional systems with complex topology [11,12]. Modelling of DCE meets well known difficulties due to description of non-stationary problems in conditions near resonance. Main analytical results was obtained here in the frameworks of "toys" onedimensional models with definite boundary movement patterns [13]. Results of applying numerical schemes to these problems may be found in $[14,15]$. Consideration of topologically nontrivial structures for realization DCE requires a number of simple analytical models for a chain of one-dimensional connected resonators with different couplings [16, 17, 19, 23-25]. Here, formalism of quantum graphs is widely used [20,26,27]. In this way, a number of spectral and transport problems were considered, particularly, with parameter-dependent or time-dependent characteristics (see, e.g., [21, 22, 28-30]).

In this paper, we generalize 1D model of the dynamical Casimir effect developed in [2-6] to take into account arbitrary variation of boundary conditions. We design a numerical scheme based on the finite element method which is simpler in implementation than the spectral decomposition used in the cited papers, especially if the boundary conditions are not trivial. Using the numerical technique, we compare photon generation due to variation of the geometry of the cavity and due to perturbation of the boundary conditions. We demonstrate that effect of the boundary conditions is significant and should be taken into account.

The paper is organized as follows. In Section 2, we recall well- known results obtained for the cavity with moving walls and field quantization inside it. Here, we also introduce changing boundary conditions (smooth switching back and forth from Dirichlet to Neumann) into the non-stationary 1D problem with moving walls and describe parameters in which dynamics will be investigated numerically. Section 3 contains description of numerical scheme, calculation methods and results of numerical modelling. In Section 4, we discuss obtained results and conclude the paper. 


\section{Electromagnetic field in a cavity with non-stationary geometry}

In this section we recall a one-dimensional problem of Casimir radiation in a cavity with moving boundaries in the same way as in [6]. In addition, we develop a formalism which includes the problem of changing the boundary conditions at the both sides of a one dimensional cavity. We start with the wave equation:

$$
\partial_{t}^{2} u(x, t)=\partial_{x}^{2} u(x, t),
$$

defined on the domain:

$$
\Omega=\{(x, t): t \in \mathbb{R},-L(t) \leq x \leq L(t)\}, \quad u: \Omega \rightarrow \mathbb{R} .
$$

On the infinities the Dirichlet boundary conditions (BC) are fixed with: $L(t)=L$ for $t<0$ and for $t>T>0$. The time dependence of the interval length $L(t)$ is assumed to be smooth. On the time interval $0 \leq t \leq T$, the $\mathrm{BCs}$ are time dependent and are given by the Robin conditions:

$$
\begin{aligned}
\alpha_{1} u(-L(t), t) & =\partial_{x} u(-L(t), t), \\
\alpha_{2} u(L(t), t) & =-\partial_{x} u(L(t), t) .
\end{aligned}
$$

As a particular case, we can deal with the Dirichlet conditions. The solution $u$ to the wave equation is assumed to be square integrable over $\Omega$.

One can observe an analogy between our procedure and the solution of 1D stationary scattering problem (if the time is considered as a spatial variable). The general solution to equation (1) for any $t$ can be decomposed over a basis of instantaneous functions $\varphi_{k}(x, t)$ :

$$
u(x, t)=\sum_{k=1}^{\infty} c_{k}(t) \varphi_{k}(x, t)
$$

where functions $\varphi_{k}(x, t)$ are eigenfunctions of the operator $\partial_{x}^{2}$ with the boundary conditions for the wave equation for fixed time $t$ (with Dirichlet BC):

$$
\partial_{x}^{2} \varphi_{k}(x ; t)=-\omega_{k}^{2}(t) \varphi_{k}(x ; t)
$$

For $t<0$ and $t>T$ the instantaneous basis is explicitly known, particularly, for the Dirichlet case it is as follows:

$$
\omega_{k}=\frac{\pi k}{L}, \quad \varphi_{k}(x ; t)=\sin \frac{\pi k x}{L}, \quad k \in \mathbb{N} .
$$

Then, the functions $c_{k}$ can be chosen in the form:

$$
\begin{cases}c_{k}(t)=a_{+, k}^{<} e^{i \omega_{k} t}+a_{-, k}^{<} e^{-i \omega_{k} t} & \text { for } t<0, \\ c_{k}(t)=a_{+, k}^{>} e^{i \omega_{k} t}+a_{-, k}^{>} e^{-i \omega_{k} t} & \text { for } \quad t>T .\end{cases}
$$

with coefficients which must form adjoint pairs:

$$
a_{+, k}^{<}=\bar{a}_{-, k}^{<}, \quad a_{+, k}^{>}=\bar{a}_{-, k}^{>} .
$$

At the infinities $t \rightarrow \pm \infty$ the decompositions over exponentials do not coincide generally, but they are related by a linear mapping (due to linearity of the wave equation):

$$
a_{+, k}^{>}=A_{k, j} a_{-, k}^{<}+B_{k, j} a_{+, k}^{<}, \quad a_{-, k}^{>}=\bar{B}_{k, j} a_{-, k}^{<}+\bar{A}_{k, j} a_{+, k}^{<} .
$$

We find the coefficients $A$ and $B$ in the case when all terms in the decomposition (3) equal zero for $t<0$ except $a_{-, k}^{<}=1$, which is:

$$
u(x, t)=\psi_{k}(x, t):=e^{-i \omega_{k} t} \varphi_{k}(x ; t), \quad t<0 .
$$

Here, $\psi_{k}(x, t)$ does not belong to $L^{2}(\Omega)$, but it satisfies boundary conditions and satisfies to the wave equation. According to (3), (4) and our assumption of $a^{<}$the following is valid for $t>T$ :

$$
\psi_{k}(x ; t)=\sum_{j=1}^{\infty}\left(a_{+, j}^{>} e^{i \omega_{j} t}+a_{-, j}^{>} e^{-i \omega_{j} t}\right) \varphi_{j}(x, t)=\sum_{j=1}^{\infty}\left(A_{k, j} e^{i \omega_{j} t}+\bar{B}_{k, j} e^{-i \omega_{j} t}\right) \varphi_{j}(x, t) .
$$

In the interval $0 \leq t \leq T$ the function $\psi_{k}(x, t)$ may be decomposed over $\varphi_{j}(x, t)$ in the same way as the function $u(x, t)$ in $(3)$ :

$$
\psi_{k}(x, t)=\sum_{j} q_{k, j}(t) \varphi_{j}(x, t)
$$

Due to (5), the following initial conditions should be satisfied:

$$
q_{k, j}(0)=\delta_{j, k}, \quad \dot{q}_{k, j}(0)=-i \omega_{k} \delta_{j, k},
$$


where $\delta_{k, j}$ is Kronecker delta. Substituting (5) into the wave equation (1), we obtain the following expression for coefficients $q_{k, j}(t)[2,6]$ :

$$
\ddot{q}_{k, j}+\omega_{j}^{2}(t) q_{k, j}=2 \lambda(t) \sum_{n} g_{j n} \dot{q}_{k, n}+\dot{\lambda}(t) \sum_{n} g_{j n} q_{k, n}+\lambda^{2}(t) \sum_{n, m} g_{n, j} g_{n, m} q_{m},
$$

where:

$$
\begin{gathered}
\partial_{x}^{2} \varphi_{j}(x, t)=-\omega_{j}^{2}(t) \varphi_{j}(x, t), \quad \omega_{j}(t)=\frac{j \pi}{L(t)} \\
g_{m j}=\frac{1}{\lambda(t)} \int_{0}^{l(t)} \partial_{t} \varphi_{j}(x, t) \cdot \varphi_{m}(x, t) d x, \quad \lambda(t)=\frac{\dot{L}(t)}{L(t)} .
\end{gathered}
$$

Therefore, $q_{k, j}$ can be computed as the solution to the obtained inhomogeneous ODE with coefficients depending on time. For varying length $L=L(t)$, but fixed boundary conditions, all contributions are known explicitly [2,6,9]:

$$
g_{j j}=0 \quad \text { and } \quad g_{k j}=(-1)^{k-j} \frac{2 k j}{j^{2}-k^{2}}=-g_{j k} \text { for } k \neq j,
$$

and analytical solution of (9) may be obtained approximately for a number of boundary motion lows [9,10]. In general, solution of this equation is expressed through hypergeometric functions and its analysis is quite cumbersome.

According to (6) and (7), $q_{k}$ is a sum of plain waves for $t>T$ :

$$
q_{k, j}(t)=A_{k, j} e^{-i \omega_{j} t}+\bar{B}_{k, j} e^{i \omega_{j} t}
$$

We will compute the scattering amplitudes $A_{k, j}$ and $B_{k, j}$ in terms of the solution $q_{k, j}$ of the ODE above:

$$
\left(\begin{array}{c}
A_{k, j} \\
\bar{B}_{k, j}
\end{array}\right)=\left(\begin{array}{cc}
e^{i \omega_{j} T} & e^{-i \omega_{j} T} \\
i \omega_{j} e^{i \omega_{j} T} & -i \omega_{j} e^{-i \omega_{j} T}
\end{array}\right)^{-1}\left(\begin{array}{c}
q_{k, j}(T) \\
\dot{q}_{k, j}(T)
\end{array}\right)
$$

To describe Casimir radiation resulting from the cavity trembling one should perform quantization of the boundary problem (1) following the standard quantization procedure as, for example, in [3]. For these, we introduce corresponding bosonic operators $\left(a^{\dagger}\right.$ is adjoint to $\left.a\right)$

$$
\begin{array}{ll}
a_{+, k}^{<}(t) \rightarrow a_{k}^{<\dagger}, & a_{-, k}^{<}(t) \rightarrow a_{k}, \\
a_{+, k}^{>}(t) \rightarrow a_{k}^{>\dagger}, & a_{-, k}^{>}(t) \rightarrow a_{k} .
\end{array}
$$

By definition of annihilation and creation operators:

$$
a_{j}^{<}|0\rangle=0, \quad a_{j}^{<\dagger}|0\rangle=|1\rangle_{j}
$$

Operators $a_{k}^{>}$and $a_{j}^{<}$are related by Bogoliubov transformation:

$$
a_{k}^{>}=\sum_{j} A_{k j} a_{j}^{<}+B_{k j} a_{j}^{<\dagger} .
$$

Mean photon number $N_{k}$ in mode $k$ and at moment $t>T$ is calculated under assumption that at time $t<0$ all modes were in a vacuum state $|0\rangle$ :

$$
N_{k}=\left\langle 0\left|a_{k}^{>\dagger} a_{k}^{>}\right| 0\right\rangle=\sum_{j}\left\langle 0\left|\left(A_{k j} a_{j}^{<}+B_{k j} a_{j}^{<\dagger}\right)^{\dagger}\left(A_{k j} a_{j}^{<}+B_{k j} a_{j}^{<\dagger}\right)\right| 0\right\rangle .
$$

therefore coefficients $A_{k j}$ disappear and we have:

$$
N_{k}=\sum_{j} B_{k j}^{*} B_{k j}\left\langle 0\left|a_{j}^{<} a_{j}^{<\dagger}\right| 0\right\rangle=\sum_{j}\left|B_{k j}\right|^{2} .
$$

\section{Numerical modelling of photon generation process}

\subsection{Numerical procedure}

The decomposition of solution to the wave equation over instantaneous basis $\varphi_{k}(x, t)$ (Fourier method), described in the previous section, was successfully used for analysis of resonator of time-dependent length, see e.g. [3,4,9, 10]. However, if the shape of the resonator or the boundary conditions evolve with time, the instantaneous basis is hard to compute explicitly and the method is not useful in practice. For numerical computations with complex geometries and complex boundary conditions the finite element method (FEM) proves to be useful in various fields of science. In the section we apply FEM to analysis of the photon generation. 
The crucial part of the computations is solution of the time-dependent wave equation (1) in the time interval $[0, T]$ with the Cauchy conditions at $t=0$. Knowing the solution at $t=T$ the computation of number of generated photon is straightforward according to (17).

Due to simple geometry, we take advantage of using the basis for all moments of time. For that purpose, we make change of the coordinate $y=x / L(t)$. Making the substitution the wave equation (1) becomes the following equation on the function $v(y)=u(y L(t))$ :

$$
a^{2} v^{\prime \prime}=L^{2} \ddot{v}-2 y \dot{L} L \dot{v}^{\prime}+y^{2} \dot{L}^{2} v^{\prime \prime}-\left(\ddot{L} L-2 \dot{L}^{2}\right) y v^{\prime}
$$

with boundary conditions:

$$
\alpha_{1}(t) L(t) v(-1)=v^{\prime}(-1), \quad \alpha_{2}(t) L(t) v(1)=-v^{\prime}(1)
$$

where:

$$
\dot{v}=d v / d t, \quad u^{\prime}=d v / d x, \quad v^{\prime}=d v / d y,
$$

and we substitute second derivatives according to:

$$
\begin{aligned}
u^{\prime}=\frac{d u}{d x}=\frac{1}{L} v^{\prime}, \quad u^{\prime \prime} & =\frac{1}{L^{2}} v^{\prime \prime}, \quad \dot{u}(t, x)=\frac{d v\left(t ; L(t)^{-1} x\right)}{d t}=\dot{v}(t, y)-v^{\prime}(t, y) \frac{y \dot{L}}{L} . \\
\ddot{u}(t, x) & =\ddot{v}-2 \dot{v}^{\prime} \frac{y \dot{L}}{L}+\left(\frac{y \dot{L}}{L}\right)^{2} v^{\prime \prime}-\left(\frac{\ddot{L}}{L}-2 \frac{\dot{L}^{2}}{L^{2}}\right) y v^{\prime} .
\end{aligned}
$$

Multiplying the equation by test function $\phi(y)$, integrating by parts and applying boundary conditions, we obtain the wave equation in the weak form:

$$
\begin{aligned}
\int_{-1}^{1}\left(L^{2} \ddot{v} \phi-2 \dot{L} L y \dot{v}^{\prime} \phi-\dot{L}^{2} y^{2} v^{\prime} \phi^{\prime}+a^{2} v^{\prime} \phi^{\prime}-\ddot{L} L y v^{\prime} \phi\right) d y & \\
& +\left(\dot{L}^{2}-a^{2}\right) L\left[\alpha_{2} v(1) \phi(1)+\alpha_{1} v(-1) \phi(-1)\right]=0 .
\end{aligned}
$$

We fix a basis $b_{k}$ in a computational subspace in $L^{2}[0,1]$, and decompose all the functions over the basis:

$$
\phi(t, y)=\sum_{n} \phi_{n}(t) b_{n}(y), \quad v(t, y)=\sum_{n} v_{n}(t) b_{n}(y) .
$$

We use basis of continuous functions which are piecewise linear on every interval $\left[y_{n}, y_{n+1}\right], y_{n}=n / N$, $n=-N \ldots N$ such that $b_{n}\left(y_{k}\right)=\delta_{n, k}$. All the elements can be expressed in term of the following canonical element $e$ :

$$
b_{n}(y)=e(n+y / \Delta), \quad \Delta=2 /(N+1), \quad e(z)= \begin{cases}1-|z|, & |z|<1 \\ 0, & |z| \geq 0\end{cases}
$$

Then equation (18) takes the form of the following system of linear equations for all $j$ :

$$
\sum_{k} L^{2} \ddot{v}_{k} A_{j k}-2 \dot{L} L \dot{v}_{k} B_{j k}-\dot{L}^{2} v_{k} D_{j k}+a^{2} v_{k} M_{j k}-\ddot{L} L v_{k} B_{j k}+\left(\dot{L}^{2}-a^{2}\right) L \delta_{j k}\left[\alpha_{1} \delta_{j,-N}+\alpha_{2} \delta_{j, N}\right]=0
$$

where the constant matrix coefficients can be computed explicitly (doing change $z=y / \Delta$ ):

$$
\begin{gathered}
A_{j k}=\int_{-1}^{1} b_{k} b_{j} d y=\Delta \int_{-N}^{N} e(z+k) e(z+j) d z=\Delta \begin{cases}\left(\delta_{k>-N}+\delta_{k<N}\right) / 3, & k=j, \\
1 / 6, & |k-j|=1, \\
0, & \text { otherwise },\end{cases} \\
B_{j k}=\int_{-1}^{1} b_{k}^{\prime} b_{j} y d y=\Delta \begin{cases}\left(\delta_{k>-N}-\delta_{k<N}\right)(1 / 6-k / 2), & k=j, \\
-1 / 3 \pm k / 2, & j=k \pm 1, \\
0, & \text { otherwise },\end{cases} \\
D_{j k}=\int_{-1}^{1} b_{k}^{\prime} b_{j}^{\prime} y^{2} d y=\Delta \begin{cases}\left(\delta_{k>-N}\left(3 k^{2}-3 k+1\right)+\delta_{k<N}\left(3 k^{2}+3 k+1\right)\right) / 3, & k=j, \\
k^{2} \pm k+1 / 3, & j=k \pm 1, \\
0, & \text { otherwise },\end{cases}
\end{gathered}
$$




$$
M_{j k}=\int_{-1}^{1} b_{k}^{\prime} b_{j}^{\prime} d y=\frac{1}{\Delta} \begin{cases}\delta_{k>-N}+\delta_{k<N}, & k=j \\ 1, & |j-k|=1 \\ 0, & \text { otherwise }\end{cases}
$$

Grouping terms for given order of derivative, we obtain:

$$
L^{2} A \ddot{v}-2 \dot{L} L B \dot{v}+\left(a^{2} M-\dot{L}^{2} D-\ddot{L} L B\right) v+\left(\dot{L}^{2}-a^{2}\right) L\left(\alpha_{1} F_{-N, j k}+\alpha_{2} F_{N}\right)=0 \quad \forall j,
$$

where non-zero entries of the matrices $F_{n}$ are only as follows: $F_{n, j k}=\delta_{n j} \delta_{n k}$. The matrices $A, B, D$ and $M$ can be precomputed, since we assume the discretization to be fixed, hence the dependence on the resonator length $L$ and the boundary conditions $\alpha_{1}, \alpha_{2}$ are explicitly stated in Equation 19. The matrix $A$ is invertible, therefore, the equation can be solved with respect to $\ddot{v}$. The order of ODE can be reduced introducing $Y=(\dot{v}, v)$, which satisfies:

$$
\begin{gathered}
Q(t) \dot{Y}=P(t) Y, \quad Q=\left(\begin{array}{cc}
L^{2} A & 0 \\
0 & 1
\end{array}\right) \\
P=\left(\begin{array}{cc}
2 \dot{L} L B & \dot{L}^{2} D-a^{2} M+\ddot{L} L B+\left(a^{2}-\dot{L}^{2}\right) L\left(\alpha_{2} F_{N}+\alpha_{1} F_{N}\right) \\
1 & 0
\end{array}\right) .
\end{gathered}
$$

We do integration over time using fifth order Radau method with three stages. The method is defined by the following Butcher table:

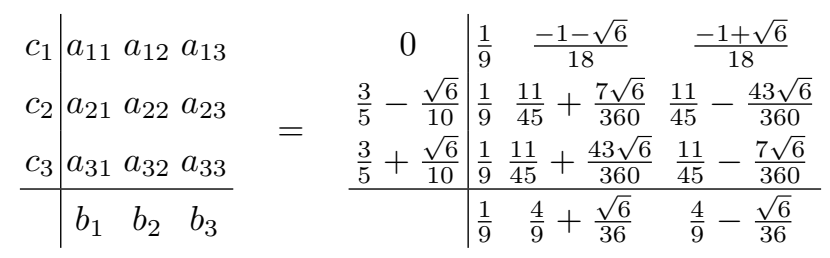

For a selected time, step $d t$, the solution $v$ is updated to the next time step according to:

$$
Y(t+d t)=Y(t)+d t \sum_{s=1}^{3} b_{s} K_{s}
$$

The vectors $K_{s}$ are solutions to the algebraic system:

$$
Q\left(t+c_{s} d t\right) K_{s}=P\left(t+c_{s} d t\right)\left[Y(t)+d t \sum_{q=1}^{3} a_{s q} K_{q}\right] \quad \forall s .
$$

All the matrices are tridiagonal, hence, block tridiagonal solver can be used to solve the system. Another option is to solve the linear system using bi-conjugate gradient method, which we adopt here (the matrix $B$ is not symmetric).

\subsection{Numerical simulation results}

The framework developed above allows us to compare effect of both cavity length and boundary conditions periodic variation on number of generated photons due to dynamical Casimir effect. The correct model of experiment in [8], where the dynamical Casimir effect was observed in a SQUID device, should probably take into account both change of the effective length and boundary conditions, however until now, theorists were focused on the length modulation. However, as we demonstrate below time-dependent boundary conditions give comparable or larger contribution to the photon generation than the time-dependent geometry.

We run simulation of the 1D cavity modeled by a segment $(-L, L)$ where the segment length is modulated according to $L(t)=1+A \sin \pi \omega_{L} t, A$ is the amplitude of oscillations (set to 0.1 in our simulations) and $\omega_{L}$ is the oscillation frequency. We use the same dimensionless units as in Equation (1) and set speed of light equals 1 . The period of the lowest eigenmode of the resonator is 2 in the unit and we consider the cavity trembling time $T$ of the same magnitude. The velocity of boundary in all the simulations was smaller than $30 \%$ of the speed of light. The interval of simulation was taken to be small multiples of the trembling time $T$. These short time intervals of transition from eigenmodes to the cavity wall induced dynamics of the electromagnetic field is hard to observe in practice, hence our simulation provides some insight into the process.

The boundary conditions are assumed to be of Robin's type (2). The parameters $\alpha_{1}, \alpha_{2}$ in the conditions oscillate from $+\infty$ (Dirichlet type) to 0 (Neumann type). A sufficiently large value of the parameters gives almost vanishing value on the boundary in the simulation, hence we restrict ourself to variation of $\alpha_{k}$ in the interval $\left[10^{-3}, 10^{3}\right]$ and its oscillation is defined as follows:

$$
\alpha_{1}=\exp \left(7 \cos \pi \omega_{\alpha} t\right)
$$


where $\omega_{\alpha}$ is the boundary condition oscillation rate. The formula is chosen somewhat arbitrary, but it guarantees symmetry between the Dirichlet and the Neumann conditions. The trembling time $T$ is always an integer multiple $K$ of switching period to ensure the Dirichlet boundary conditions in the beginning and the end of the trembling time interval, that is $T=2 \mathrm{~K} / \omega_{a}$.

The solution to the time-dependent wave equation are shown in Fig. 1 for varying length $L$ and in Fig. 2 for varying boundary conditions. The effect of variation in both cases are stronger for higher cavity trembling frequency. For the frequency $\omega_{L}$ of the cavity wall motion few times smaller than ground mode of the cavity, the evolution is adiabatic and the solution $u$ is only slightly affected by the geometry modification. The cavity wall moving faster generates waves and gradually eliminate the ground mode, resulting in Helmholtz-like motion having triangle pattern in Fig. 1, which repeats itself infinitely.
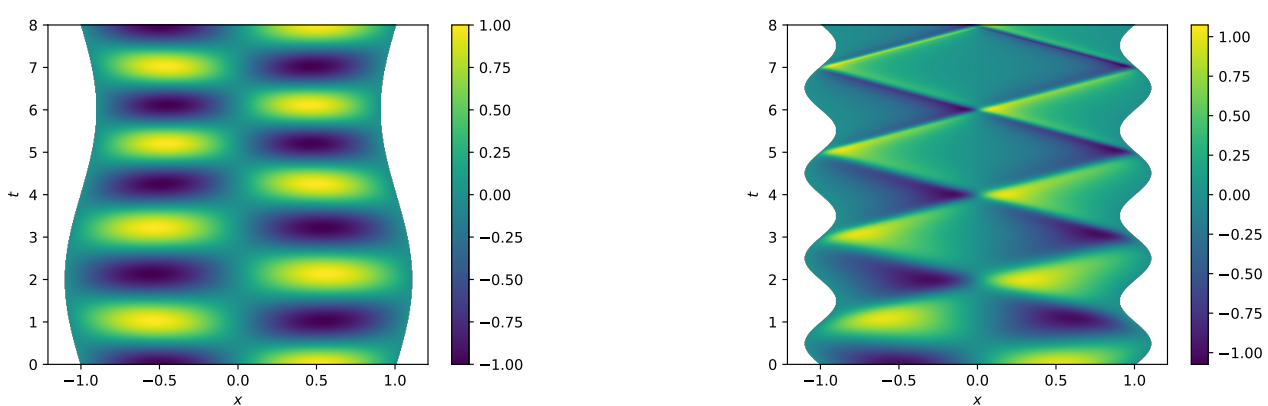

FIG. 1. Evolution of the ground mode of the cavity according to the wave equation with moving boundary for the simulation interval length $T=8$ : (left) adiabatic regime $\omega_{L}=0.25$, (right) the cavity wall driven regime $\omega_{L}=1$. Heat map shows real part of the solution.
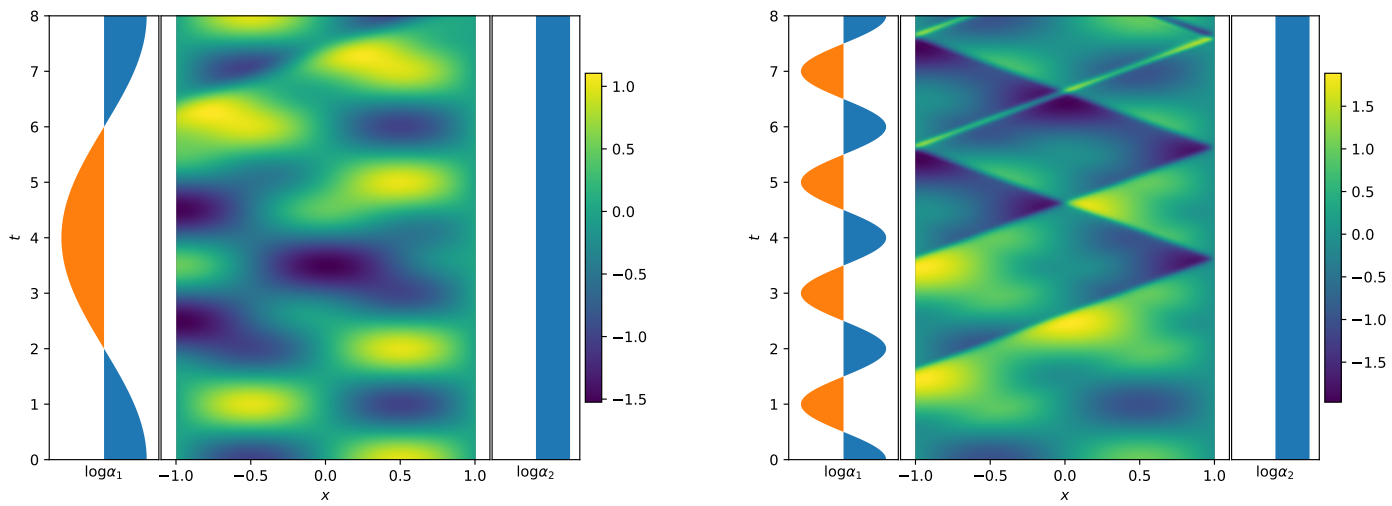

FIG. 2. Solution $u$ to the wave equation with oscillating Robin's boundary condition for the simulation interval length $T=8$ : (left) low frequency regime $\omega_{\alpha}=0.25$, (right) the cavity wall driven regime $\omega_{\alpha}=1$. Initial conditions correspond to the resonator in the ground state. Heat map shows real part of the solution. The Robin's boundary conditions parameters are shown on left $\left(\log \alpha_{1}\right)$ and right $\left(\log \alpha_{2}\right)$ panels, initial conditions correspond to the Dirichlet $\mathrm{BC}$, negative values corresponds to the Neumann BC.

The variation of boundary conditions results in change of eigenfrequencies of the resonator, hence noticeably affects dynamics even for small frequencies $\omega_{\alpha}$. Since we assume time-dependent boundary conditions on single boundary, the symmetry of solution is broken, thus the irregular texture on the heat map. The amplitude of the solution is not conserved in the process, hence number of generated photons is expected to be larger for longer generation period. For rapid boundary conditions switching, the ground state rapidly dissipates on the boundary, and the solution to the wave equation is dominated by wave with source on the boundary of the cavity. 
We analyzed 20 lowest modes of the resonator using 201 finite element doing 4th order Runge-Kutta simulation with time step $4 \cdot 10^{-4}$. Then, the average number of photons generated due to the dynamical Casimir effect was estimated according to Equation (17). The computation was repeated for different periods of boundary oscillations, the result are presented in Fig. 3. We computed total number of generated photons for boundary oscillation repeated $K$ times, $K \leq 3$. In all cases, the number of generated photons decreases as trembling time $T$ increases (and therefore oscillation frequency tends to zero). However, the decrease is not monotonic and is quite intricate. The decrease is slower for the variation of the boundary conditions, and total number of photons tends to be smaller for the oscillation of the cavity size.
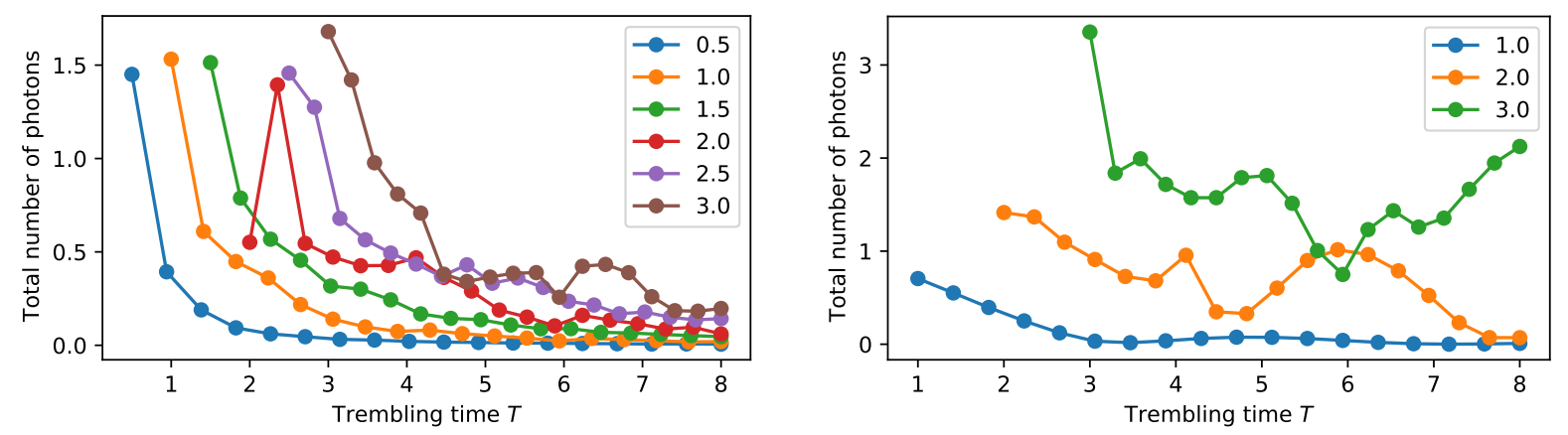

FIG. 3. Average number of generated photons as function of length $T$ of time interval when perturbation to the boundary is applied. Left panel: the cavity length $L$ is varied, right panel: the boundary conditions parameter $\alpha_{1}$ is oscillating. Perturbation frequency $\omega=2 K / T$ is matched with $T$ in such a way that $K$ period of oscillation happen during time $T$. Different lines correspond to different values of $K$.

The lines with higher $K$ illustrates what happens under repetition of the boundary perturbation. E.g. point for $K=2$ and $T=2$ correspond to double repetition of the oscillation for $K=1$ and $T=1$. As we see from the left panel in Fig. 3, repetition of the cavity shrinking does not produce more photons then the single perturbation, except for few points. On the contrary, the repetition of oscillation of the boundary conditions do produce new photons, even more the dependence of number of photons on number of repetitions $K$ is faster than linear.

\section{Discussion and conclusion}

We considered generalized model of dynamic Casimir effect in a trembling cavity taking into account periodic perturbation of boundary conditions. The numerical scheme described in the article for solution of the 1D wave equation with time-dependent geometry and boundary conditions forms a basis for simulation of dynamical Casimir effect relaxing restrictions on the behavior of the boundary. Our numerical simulations demonstrated that boundary conditions oscillation has significant effect on number of generated photons and the number can be even larger than for variation of effective length of the resonator.

The developed numerical scheme based on finite element method can be naturally extended to two and three dimensional cavities, and can be used to simulate experimental settings. Since perturbation of the boundary conditions can be used to boost photon generation, quantum graphs (also called quantum networks) is a natural candidate to increase effectiveness of the generation process.

\section{Acknowledgements}

The review of quantum non-stationary, multi-mode parametric processes and dynamical Casimir effect in Section 2 was supported by the Ministry of Science and Education of the Russian Federation (Passport No. 2019-0903). Investigation of 1D cavity with moving walls in Section 2 was partially financially supported by grant 20-31-90036 of Russian Foundation for Basic Research. The development of numerical method of simulation of dynamical Casimir effect in Section 3 was funded by Russian Science Foundation (Grant 19-42-06302). 


\section{References}

[1] Nation P.D., Johansson J.R., Blencowe M.P. Colloquium: Stimulating uncertainty: Amplifying the quantum vacuum with superconducting circuits. Rev. Mod. Phys., 2012, 84, P. 1-24.

[2] Crocce M. Resonant photon creation in a three-dimensional oscillating cavity. Phys. Rev. A, 2001,64 (1), 013808.

[3] Law C. Effective hamiltonian for the radiation in a cavity with a moving mirror and a time-varying dielectric medium. Physical Review A, 1994, 49 (1), P. 433-437.

[4] Law C. Interaction between a moving mirror and radiation pressure: A hamiltonian formulation. Physical Review A, 1995, 51 (3), P. 25372541.

[5] Sassaroli E., Srivastava Y., Widom A. Photon production by the dynamical casimir effect. Phys. Rev. A, 1994, 50 (2).

[6] Dodonov V. Generation and detection of photons in a cavity with a resonantly oscillating boundary. Phys. Rev. A, 1996, 53 (4).

[7] Moore G. Quantum theory of the electromagnetic field in a variable length one dimensional cavity. Journal of Mathematical Physics, 1970, 11, P 2679.

[8] Wilson C., Johansson G. Observation of the dynamical Casimir effect in a superconduction circuit. Nature, 2011, 479, P. 376-379.

[9] Dodonov V. Current status of the dynamical Casimir effect. Physica Scripta, 2010, 82, 038105.

[10] Dodonov V.V. Fifty years of the dynamical Casimir effect. Physics, 2020, 2 (3), P. 67-104.

[11] Lähteenmäki P., Paraoanu G.S., Hassel J., Hakonen P.J. Dynamical Casimir effect in a Josephson metamaterial. Proceedings of the National Academy of Sciences, 2013, 110 (11), P. 4234-4238.

[12] Svensson I.-M., Pierre M., et al. Microwave photon generation in a doubly tunable superconducting resonator. Journal of Physics: Conference Series, 2018, 969, 012146.

[13] Good M.R.R., Linder E.V. Slicing the vacuum: New accelerating mirror solutions of the dynamical Casimir effect. Phys. Rev. D, 2017, 96, 125010.

[14] Li L., Li B.-Z. Numerical solutions of the generalized Moore's equations for a one-dimensional cavity with two moving mirrors. Physics Letters A, 2002, 300 (1), P. 27-32.

[15] Alves D.T., Granhen E.R. A computer algebra package for calculation of the energy density produced via the dynamical casimir effect in one-dimensional cavities. Computer Physics Communications, 2014, 185 (7), P. 2101-2114.

[16] Popov I.Y. Asymptotic series for the spectrum of the Schrödinger operator for layers coupled through small windows. Theor. Math. Phys., 2002, 131 (3), P. 407-418.

[17] Popov I.Y. Asymptotics of bound states and bands for laterally coupled waveguides and layers. J. Math. Phys., 2002,43 (1), P. $215-234$.

[18] Popov I., Trifanov A.I., Trifanova E.S. Coupled dielectric waveguides with photonic crystal properties. Zh. Vychisl. Mat. Mat. Fiz., 2010, 50, P. 1931-1937.

[19] Nekrashevych V., Teplyaev A. Groups and analysis on fractals, analysis on graphs and its applications. Amer. Math. Soc., 2009, 77, P. 143-180.

[20] Korotyaev E. Schrodinger operator with a junction of two 1-dimensional periodic potentials. Asymptotic Analysis, 2005 , 45, P. 73-97.

[21] Matrasulov D.U., Yusupov J.R., Sabirov K.K., Sobirov Z.A. Time-dependent quantum graph. Nanosystems: Phisycs, Chemistry, Mahematics, 2015, 6 (2), P. 173-181.

[22] Lobanov I.S., Nikiforov D.S., et al. Model of time-dependent geometric graph for dynamical Casimir effect. Indian Journal of Physics, 2020, DOI 10.1007/s12648-020-01866-5.

[23] Popov I.Yu., Trifanov A.I., Trifanova E.S. Dielectric waveguides with photonic crystal properties. Comp. Math. Math. Phys., 2010, 50 (11), P. 1830-1836.

[24] Melikhova A.S., Popov I.Y. Spectral problem for solvable model of bent nanopeapod. Applicable Analysis, 2017,96 (2), P. $215-224$.

[25] Vorobiev A.M., Bagmutov A.S., Popov A.I. On formal asymptotic expansion of resonance for quantum waveguide with perforated semitransparent barrier. Nanosystems: Phys. Chem. Math., 2019, 10 (4), P. 415-419.

[26] Exner P., Kostenko A., Malamud M., Neidhardt H. Spectral theory of infinite quantum graphs. Ann. H. Poincare, 2018, 19, P. 3457-3510.

[27] Berkolaiko G., Kuchment P. Introduction to Quantum Graphs. AMS, Providence, 2012.

[28] Eremin D.A., Grishanov E.N., Nikiforov D.S., Popov I.Y. Wave dynamics on time-depending graph with Aharonov-Bohm ring. Nanosystems: Physics, Chemistry, Mathematics, 2018, 9 (4), P. 457-463.

[29] Barseghyan D., Exner P., Khrabustovskyi A., Tater M. Spectral analysis of a class of Schrödinger operators exhibiting a parameter-dependent spectral transition. J. Phys. A, 2016, 49, 165302.

[30] Fedorov E.G., Popov A.I., Popov I.Y. Metric graph version of the FitzHugh-Nagumo model. Nanosystems: Physics, Chemistry, Mathematics, 2019, 10 (6), P. 623-626. 